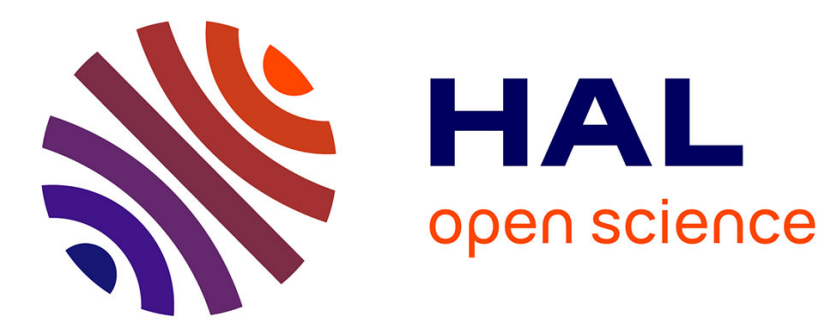

\title{
Dynamic Bayesian Networks modelling the dependability of systems with degradations and exogenous constraints
}

Philippe Weber, Lionel Jouffe, Paul Munteanu

\section{- To cite this version:}

Philippe Weber, Lionel Jouffe, Paul Munteanu. Dynamic Bayesian Networks modelling the dependability of systems with degradations and exogenous constraints. Apr 2004, pp.cd-rom. hal-00131316

\author{
HAL Id: hal-00131316 \\ https://hal.science/hal-00131316
}

Submitted on 16 Feb 2007

HAL is a multi-disciplinary open access archive for the deposit and dissemination of scientific research documents, whether they are published or not. The documents may come from teaching and research institutions in France or abroad, or from public or private research centers.
L'archive ouverte pluridisciplinaire HAL, est destinée au dépôt et à la diffusion de documents scientifiques de niveau recherche, publiés ou non, émanant des établissements d'enseignement et de recherche français ou étrangers, des laboratoires publics ou privés. 
WEBER P., MUNTEANU P., JOUFFE L. Dynamic Bayesian Networks modelling the dependability of systems with degradations and exogenous constraints. 11th IFAC Symposium on Information Control Problems in Manufacturing, INCOM'04. Salvador-Bahia, Brazil, April 5-7th, 2004.

\title{
DYNAMIC BAYESIAN NETWORKS MODELLING THE DEPENDABILITY OF SYSTEMS WITH DEGRADATIONS AND EXOGENOUS CONSTRAINTS
}

\author{
Weber P. ${ }^{+}$, Munteanu P.*, Jouffe L. \\ ${ }^{+}$Centre de Recherche en Automatique de Nancy (CRAN), CNRS UMR 7039 \\ Université Henri Poincaré, Nancy 1; 2, rue Jean Lamour \\ 54519 VANDOEUVRE-LES-NANCY Cedex - France, \\ (Philippe.Weber@esstin.uhp-nancy.fr) \\ * Centre de Recherche du Groupe ESIEA \\ 38, rue des Docteurs Calmette et Guérin \\ 53000 LAVAL - France, (\{munteanu,jouffe\}@esiea-ouest.fr)
}

\begin{abstract}
The work reported here presents an original method to model dependability of systems, taking into account degradations and failure modes governed by exogenous constraints. The component degradation dynamics is considered as a semi-Markov process. Environmental behaviour introduces switching models conditioned by exogenous constraints. Dynamic Bayesian Networks (DBN) are employed to formalise such complex dynamic processes through a compact representation. DBN allow simulating these processes, taking into account events due to the environmental behaviour. A hydraulic system is used to illustrate the reliability estimations obtained by the proposed modelling method
\end{abstract}

Keywords: Dynamic Bayesian Network, Markov Switching Model, Hidden Markov Models, Reliability evaluation.

\section{INTRODUCTION}

One of the main challenges of the extended enterprise is to dynamically maintain and optimise the quality of the services delivered by industrial processes along their life cycle. The goal is to design decision-making aid systems to maintain these processes in operation. Nevertheless, most of the current automated systems do not provide the means for intelligent interpretation of information coping with large process disturbances. Moreover, decisions can be taken without a perfect perception of the system state. This partial perception argues in favour of using a probabilistic estimation of the system state. As described in (Boutillier, et al., 1999), tools issued from Artificial Intelligence can be used to provide decision-making aid for manufacturing systems.

Moreover, works on system dependability and Bayesian Networks (BN) have recently been developed (Kang and Golay, 1999). Bobbio, et al., (2001), explain how the Fault Tree (FT) can be achieved using BN. Torres-Toledano and Sucar (2003) present the relation between Reliability Block
Diagram (RBD) and BN. Weber, et al. (2001), proposed a model based decision system allowing fault diagnosis using system functioning and dysfunctioning analyses based on Object Oriented Bayesian Network (OOBN). The solutions proposed in these papers are all based on static probabilistic models.

In Aven and Jensen, (1999), a system made of $n$ components with positive random lifetimes is called a complex system if two or more components can fail at the same time with positive probability, and the system, as well as the components, are allowed to have an arbitrary (finite) number of states or level (in the following: a multistate system or component). The reliability estimation of such complex systems, taking into account the effects of failure combinations, results in developing more and more scenarios with respect to the system complexity. Then, the classical modelling methods are close to their limits. Moreover, in order to improve the decision-making during the diagnosis and the maintenance strategies, the goal is to define a dynamic model of the process behaviour. This model 
allows computing state probability distributions (prognosis) by taking into account the component age, the last executed maintenance operations and the evolution of the environment of the process.

Methods relying on Markov Chains (MC) models lead to a combinatorial explosion of the number of states to specify the system. This modelling method is then unsuitable for complex systems and alternative methods have been proposed. Meshkat et al., (2002) present the relation between MC and Dynamic Fault Trees (DFT). Another method, based on the DFT, is presented by Bouissou and Bon (2003). These authors introduced a modelling formalism that combines concepts from fault trees and $\mathrm{MC}$ in the formalism of Boolean logic Driven Markov Processes. Besides, we have shown that probabilistic models such as the Dynamic Bayesian Networks (DBN) allow decreasing effect of combinatorial explosion in reliability estimation by a more synthetic description of the system (Weber and Jouffe 2003).

The aim of this paper is to use DBN as an equivalent model to the MC (Padhraic, 1997). The problems considered here are those whose dynamics can be modelled as stochastic processes, and, where the decision maker's actions influence the system behaviour. The current system state and the applied action jointly determine the probability distribution over the next states.

The paper is divided into five sections. Section 2 presents the problem statement concerning the MC model and the fast growing of its state cardinality with respect to the modelled system complexity. Section 3 introduces the BN theory and defines the DBN used in the following. The proposed methodology is an original formalisation of the system reliability modelling. A simulation with a hydraulic system is developed in section 4. Finally, section 5 presents conclusions and perspectives.

\section{PROBLEM STATEMENT}

In order to take the uncertainty into account, the process behaviour is represented as a random variable that takes its values from a finite state space corresponding to the possible process states. A MC allows modelling the dynamics of sequences taken by these states (Boutillier, et al., 1999).

\subsection{The Markov Chain notations}

Let $X$ be a discrete random variable that models a process with a finite number of mutually exclusive states $\left\{s_{1}, \ldots, s_{M}\right\}$. The vector $\pi$, then, denotes a probability distribution over these states: $\pi=\left[\begin{array}{lllll}\pi\left(s_{1}\right) & \ldots & \pi\left(s_{m}\right) & \ldots & \pi\left(s_{M}\right)\end{array}\right], \pi\left(s_{m}\right) \geq 0$

with $\pi\left(s_{m}\right)=p\left(X=s_{m}\right)$ and $\sum_{m=1}^{M} \pi\left(s_{m}\right)=1$
Assuming that the system evolves between states, where the occurrence of an event marks the transition from a state $(k)$ to the next state $(k+1)$, then the process produces the sequence $\left(\pi_{0}, \pi_{1}, \ldots \pi_{k-1}, \pi_{k}\right)$ that can be modelled as a discrete MC if:

$$
\begin{aligned}
\pi_{k}\left(s_{m}\right) & =p\left(X_{k}=s_{m} \mid \pi_{0}, \pi_{1}, \ldots \pi_{k-1}\right) \\
& =p\left(X_{k}=s_{m} \mid \pi_{k-1}\right)
\end{aligned}
$$

The Markov property makes it possible to specify the statistical relationships among states as a transition probability matrix $\mathbf{P}_{M C}$. If the transition probabilities $p_{i j}=p\left(X_{k}=s_{j} \mid X_{k-1}=s_{i}\right)$ are time independent then the MC is said to be homogeneous.

$\mathbf{P}_{M C}\left(X_{k} \mid X_{k-1}\right)=\left[\begin{array}{cccc}p_{11} & p_{12} & \cdots & p_{1 M} \\ p_{21} & & & \cdots \\ \cdots & & & \cdots \\ p_{M 1} & p_{11} & \cdots & p_{M M}\end{array}\right]$

The sequence of the probability distribution is estimated by:

$$
\pi_{k}=\pi_{0}\left(\mathbf{P}_{M C}\right)^{k}
$$

\subsection{Models of system reliability}

The reliability of systems or component is classically modelled with MC. This method leads to a graphical representation (Ansell and Phillips (1994) pp. 124). Nevertheless the complexity of the model depends on the assumptions made to approximate the stochastic process. Unfortunately, the modelling complexity increases dramatically as we want to be close to the real behaviour of the system reliability. Indeed, the state space describing the system is made of the Cartesian product of the component states.

\section{Hypothesis of a Markov process}

Let's consider the reliability of a component. It is modelled by a discrete random variable $X$ with states $\{o, f\}$, in which $(o)$ indicates an operational state and $(f)$ indicates a failure state.

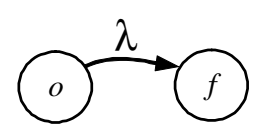

Fig. 1. Markov Chain.

To model the reliability, the matrix $\mathbf{P}_{M C}$ between the states $\{o, f\}$ is defined as follow:

$$
\mathbf{P}_{M C}=\left[\begin{array}{cc}
1-\lambda & \lambda \\
0 & 1
\end{array}\right]
$$


where $\lambda$ represents the constant failure rate and the density function of the failure time $f(t)$ is:

$\lambda(t) \equiv \frac{f(t)}{R(t)}=\frac{1}{M T T F}, f(t) \equiv-\frac{d R(t)}{d t}=\lambda e^{-\lambda t}$

where MTTF represents the Mean Time To Failure.

The solution of this process is well known and leads to the reliability $R(t)$ of a component with exponential distribution of failure time:

$$
R(t) \equiv \exp \left\{-\int_{0}^{t} \lambda(\tau) d \tau\right\}=e^{-\lambda t}
$$

From the MC, the component reliability is given by:

$$
\begin{aligned}
& R(k)=p\left(X_{k}=o \mid \pi_{k-1}\right)=\pi_{k}(o) \\
& \bar{R}(k)=p\left(X_{k}=f \mid \pi_{k-1}\right)=\pi_{k}(f)
\end{aligned}
$$

The simulation of this behaviour is defined by the recurrence deduced from eq. (4). This equation is justified by systems composed from $n$ multistate components. The complexity of the expression describing the system reliability $R_{s y s}(t)$ quickly increases with respect to the number of system states. Then eq. (4) estimates the probability distribution over the system states. Assuming that $i \in\{0 . . . l\}$ represents the functioning states of the system, system reliability is defined as:

$$
R(k)=\sum_{i \in\{0 \ldots l\}} \pi_{k}\left(s_{i}\right)
$$

\section{Hypothesis of a semi-Markov process}

When one wants to take into account degradation, the failure rate has to vary over time. This hypothesis is indeed more realistic because component aging implies more and more maintenance actions. To model this behaviour, a generalisation of the Markov Process called Semi-Markov Process (SMP) is introduced (Gertsbakh (2000) pp. 117). The classical way is to model the probability density function of the failure time distribution with a continue distribution as Gamma, Weibull or Normal (Gaussian) distributions. The matrix defined in eq.(5) becomes time dependant.

$\mathbf{P}_{M C}\left(X_{k} \mid X_{k-1}\right)=\left[\begin{array}{cc}1-\lambda(k) & \lambda(k) \\ 0 & 1\end{array}\right]$

The MC is then not homogeneous anymore. In order to model SMP, an approximation can be made using Hidden Markov Model (HMM). HMM simulates the behaviour of the probability distribution over the system states. The method consists in increasing the number of system states to model the dynamics or degradations. Then a component is modelled by a discrete random variable $X_{k}$ with states $\left\{s_{o}, s_{d 1}, s_{d 2}, \ldots s_{f}\right\}$ where $s_{d \mathrm{i}}$ models the hidden states of degradation. This modelling results in failure rate as a function of the time $\lambda(k)$. The HMM can be defined from constant parameters $\lambda_{\mathrm{ij}}$ of a generalized Erlang equivalent model (Cox 1962) (Fig. 2).

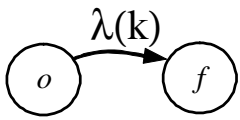

The equivalent HMM:

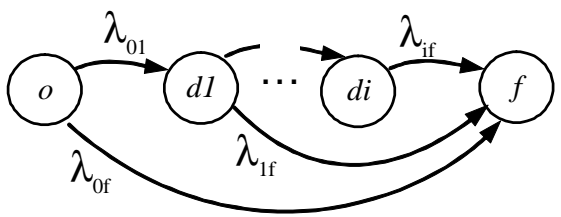

Fig. 2. Hidden Markov Model.

Therefore generalized Erlang density function approximates failure time of SMP. To estimate the reliability of the system the following equation is computed from (4):

$$
R(t)=\sum_{s_{i} \neq f} \pi_{k}\left(s_{i}\right)
$$

Hypothesis of a semi-Markov process with exogenous constraint

The system environment (exogenous events) impacts on the probability density function of the failure time distribution. Let's consider several system functioning modes. The component degradation follows then several behaviors. To take into account these exogenous events, one has to use several models to represent each situation according to an environmental context, and models become more and more complex when the system is composed by $n$ components with several failure modes.

Markov Switching Models (MSM) are introduced to model this kind of stochastic process with exogenous events. These models are also viewed as conditional HMM where the transition distributions are conditional to an exogenous variable. The MSM models the non-stationary that are due to abrupt changes in the functioning modes of the system (Bengio 1999). Furthermore, the model is represented by Input-Output HMM (IOHMM) if the distribution over the states of the exogenous variable is known. The difference between standard HMM and MSM or IOHMM is that HMM represents the distribution of $p\left(X_{k}\right)$ whereas the MSM or IOHMM represent the conditional distribution $p\left(X_{k} \mid Q^{T}{ }_{k}\right)$ given the input sequence $Q^{T}{ }_{k}=\mathrm{q}_{o}, \mathrm{q}_{1}, \ldots, \mathrm{q}_{k}$. where $\mathrm{q}_{k}$ represents the exogenous constraint with states $\left\{s_{1}, s_{2}, \ldots s_{M}\right\}$.

The computation of $\pi_{k}$ with MSM can be carried out only by approximate methods, i.e. simulation. Indeed, it is unfeasible to obtain the analytic solution of this kind of hybrid differential system. In case of IOHMM, it is the distribution over the states of the exogenous variable which condition the solution. The 
exact resolution of this model is then also unfeasible using classical MC and recurrence equation.

The MC, HMM, MSM or IOHMM are methods well suited to model the reliability of a complex entity/system of low dimension. However, within the framework of general complex systems, the combinatorial explosion of states makes these methods unmanageable. In the following, a method based on DBN is presented. It provides a synthetic representation for modelling complex systems.

\section{BAYESIAN NETWORK MODELLING}

BN are probabilistic networks based on graph theory. Each node represents a variable and arcs indicate direct probabilistic relations between the connected nodes. DBN allow taking time into account by defining different nodes to model the same variable with respect to different time slices.

\subsection{The Bayesian Network notations}

$\mathrm{BN}$ are directed acyclic graphs (Jensen, 1996) defined as a pair: $C_{l}=((N, A), \mathscr{P})$, where " $N$ " is a set of nodes; " $A$ " is a set of arcs; $\mathscr{P}$ represents the set of conditional probability distributions that quantify the probabilistic dependencies.

A discrete random variable $X$ is represented by a node $n \in N$ with a finite number of mutually exclusive states: $\mathcal{S}_{n}:\left\{s_{1}^{n}, \ldots s_{M}^{n}\right\}$. The vector $x^{n}=\left[\begin{array}{lll}p_{1} & \ldots & p_{M}\end{array}\right]$ denotes a probability distribution over $\mathcal{S}_{n}$ like eq. (1), where $p_{m}$ is the marginal probability of $n$ being in state $s_{m}^{n}$. In Fig. 3, the nodes $n_{i}$ and $n_{j}$ are linked by an $\operatorname{arc}\left(n_{i}, n_{j}\right) \in A$ then $n_{i}$ is considered as a parent of $n_{j}$. The parent set of a node $n_{j}$ contains all the parents of $n_{j}$ (e.g., $p a\left(n_{j}\right)=\left\{n_{i}\right\}$ in Fig. 3).

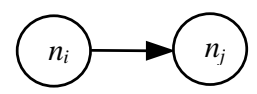

Fig. 3. A basic BN.

In this work, the set $\mathscr{P}$ is represented with Conditional Probability Tables (CPT). Then, each node has an associated CPT. For instance, in Fig. 3, $n_{i}$ and $n_{j}$ are defined over the states $\mathcal{S}_{n_{i}}:\left\{s_{1}^{n_{i}}, \ldots s_{M}^{n_{i}}\right\}$ and $\mathcal{S}_{n_{j}}:\left\{s_{1}{ }_{1}, \ldots s_{L}{ }^{n_{j}}\right\}$. Then, the CPT of $n_{j}$ is defined by the conditional probabilities $p\left(n_{j} \mid p a\left(n_{j}\right)\right)$ over each state of $n_{j}$ and of its parents. This CPT is represented as a matrix. For instance, the CPT of node $n_{j}$ of Fig. 3 is:

$$
\begin{aligned}
& \mathbf{P}\left(n_{j} \mid n_{j}\right)= \\
& {\left[\begin{array}{ccc}
p\left(n_{j}=s_{1}^{n_{j}} \mid n_{i}=s_{1}^{n_{i}}\right) & \cdots & p\left(n_{j}=s_{L}^{n_{j}} \mid n_{i}=s_{1}^{n_{i}}\right) \\
\vdots & \vdots \\
p\left(n_{j}=s_{1}^{n_{j}} \mid n_{i}=s_{M}^{n_{i}}\right) & \cdots & p\left(n_{j}=s_{L}^{n_{j}} \mid n_{i}=s_{M}^{n_{i}}\right)
\end{array}\right]}
\end{aligned}
$$

For the root nodes, i.e. the nodes without parent, the CPT contains just a row describing the a priori probability of each state.

Various algorithms can be used to do exact or approximate inference, i.e. for the computation of marginal probabilities. The most classical exact inference algorithm relies on the use of a junction tree (more explications can be found in (Jensen, 1996, pp. 76)). Inference in $\mathrm{BN}$ allows taking into account any state variable observation (an event) for the updating of the probabilities of each variable. Without observations, the computation is based on $a$ priori probabilities. As observations are made, the knowledge is incorporated in the network and the probabilities over the process states are updated.

\subsection{Dynamic Bayesian Network}

A DBN includes a temporal dimension. This new dimension is managed by time-indexed random variables. $X_{i}$ is represented at time step $k$ by a node $n_{(i, k)} \in N$ with a finite number of states $\mathcal{S}_{n_{i}}:\left\{s_{1}^{n_{i}}, \ldots s_{M}^{n_{i}}\right\} . \quad x_{k}^{n_{i}}$ denotes the probability distribution over these states at time step $k$. Several time stages are represented by several sets of nodes $N_{0}, \ldots N_{k} \cdot N_{k}$ includes all the random variables relative to the time slice $k$ (Hung, et al., 1999; Boutillier, et al., 1999, pp. 38-45).

An arc linking two variables belonging to different time slices represents a temporal probabilistic dependence between these variables. Then DBN allow to model random variables and their impacts on the future distribution of other variables. Defining these impacts as transition-probabilities between the states of the variable at time step $k$ and time step $k+1$, these transition-probabilities lead to define CPT relative to inter-time slices, equivalent to CPT defined in the previous section (eq. (12)). With this model, the future $(k+1)$ is conditionally independent of the past given the present $(k)$, which means that the CPT $\mathbf{P}\left(n_{i, k+1} \mid p a\left(n_{i, k+1}\right)\right)$ respects the Markov properties (Kjaerulff, 1995). Moreover, this CPT is equivalent to the $\mathrm{MC}$ model of the variable $X_{i}$ described in the section 2.1 if $p a\left(n_{i, k+1}\right)=n_{i, k}$ and $\mathcal{S}_{n_{i, k}}=\mathcal{S}_{n_{i, k+1}}: \mathbf{P}\left(n_{i, k+1} \mid n_{i, k}\right)=\mathbf{P}_{M C}$. Starting from an observed situation at time step $k=0$, the probability distribution $x_{k}^{n_{i}}$ over $X_{i}$ states is computed by inference. To compute $x_{k+T}^{n_{i}}$, several solutions are proposed in the literature. One of them consists in 
developing $T$ time slices (Fig. 4), obtaining then a network which size grows proportionally to $T$ (Kjaerulff, 1995). This solution is unsuitable for reliability analysis because the process have to be analysed over a large time horizon. This leads to an explosion of the number of nodes. Nevertheless, this modelling is exactly equivalent to the model described in the above section (Bengio, 1999).

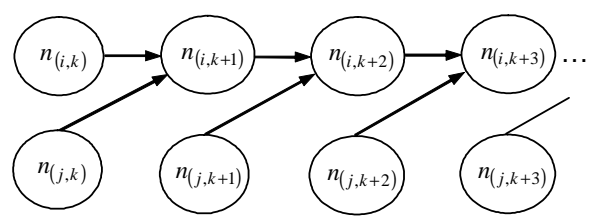

Fig. 4. A un-rollup DBN modelling IOHMM.

Another solution, which keeps a compact network form, is based on iterative inferences. This solution is used in the following. Indeed, it is possible to compute the probability distribution of any variable $X_{i}$ at time step $k+1$ based on the probabilities corresponding to time step $k$. The probability distributions at time step $k+2 \ldots$ are computed using successive inferences (Welch and Thelen 2000). Then a network called 2-TBN (Boyen and Koller 1998) (Fig. 5) with only two time slices is defined. The first slice contains the nodes corresponding to the current time step $(k)$, the second one those of the following time step $(k+1)$. A node is called temporal node when it has a direct link with its temporal clone at time step $(k+1)$, as $n_{(i, k)}$ in Fig. 5.

Observations, introduced as hard evidence or probability distributions, are only realised in the current time slice $k$. Then an introduction of the context as exogenous random variable is made by the means of a node $n_{(j, k)}$ which governs the behaviour of $X_{i}$ thank to the CPT defined in eq. (13). When all the temporal nodes are independent, we can still use an exact inference algorithm. Otherwise, we have to use approximate inference methods, as for example particle filtering (Doucet et al., 2000, Koller and

Lerner 2001). When exact inference can be used, the time increment is carried out by setting the computed marginal probabilities of the node at time step $k+1$ as The CPT is defined as $\mathbf{P}\left(n_{i, k+1} \mid n_{i, k}, n_{j, k}\right)$ :

$$
\mathbf{P}\left(n_{i, k+1} \mid n_{i, k}, n_{j, k}\right)=\left[\begin{array}{ccc}
p\left(n_{j}=s_{1}^{n_{j}} \mid n_{i}=s_{1}^{n_{i}}, n_{j}=s_{1}^{n_{j}}\right) & \cdots & p\left(n_{j}=s_{L}^{n_{j}} \mid n_{i}=s_{1}^{n_{i}}, n_{j}=s_{1}^{n_{j}}\right) \\
\vdots & \vdots \\
p\left(n_{j}=s_{1}^{n_{j}} \mid n_{i}=s_{M}^{n_{i}}, n_{j}=s_{1}^{n_{j}}\right) & \cdots & p\left(n_{j}=s_{L}^{n_{j}} \mid n_{i}=s_{M}^{\left.n_{i}, n_{j}=s_{1}^{n_{j}}\right)}\right. \\
\vdots & \\
p\left(n_{j}=s_{1}^{n_{j}} \mid n_{i}=s_{M}^{n_{i}}, n_{j}=s_{M^{\prime}}^{n_{j}}\right) & \cdots & p\left(n_{j}=s_{L}^{n_{j}} \mid n_{i}=s_{M}^{n_{i}}, n_{j}=s_{M^{\prime}}^{n_{j}}\right)
\end{array}\right]
$$

observations for its corresponding node in the previous time slice.

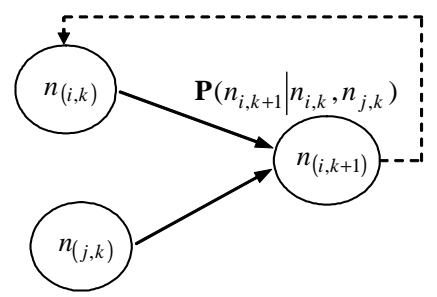

Fig. 5. A 2-TBN modelling IOHMM with temporal node $n_{(i, k)}$ and exogenous observations $n_{(j, k)}$.

\section{APPLICATION}

The proposed method is applied to a classical example of reliability analysis. This example allows comparing the method based on DBN with the one based on MC.

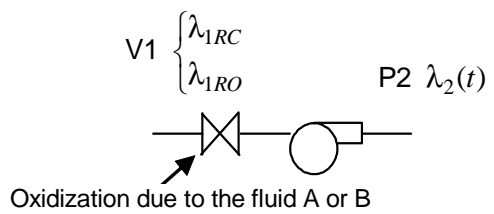

Fig. 6. Hydraulic system.

Fig. 6 describes the system. A valve V1 and a pump $\mathrm{P} 2$ are used to distribute a fluid. The valve has two failure modes: remains closed (fRC) or remains opened (fRO). The valve failure rates are the following: $\lambda_{1 R C}=1 \cdot 10^{-4} ; \lambda_{1 R O}=1.5 \cdot 10^{-4}$ and the failure rate of the pump is time dependent. This dependence follows the Weibull distribution:

$\lambda_{2}(t)=\frac{\beta^{\prime} \cdot t^{\beta^{\prime}-1}}{\eta^{\prime \beta^{\prime}}}, \beta^{\prime}=1.5, \eta^{\prime}=1000$

Parameters of the Weibull distribution are independent of the nature of the fluid. But the valve's parameters are modified by the nature of the fluid. The PH induces oxidation of the valve V1. The parameter $\lambda_{1 R O}$ and $\lambda_{1 R C}$ are modified with factor $\phi=1$ for the fluid A and $\phi=4$ the fluid B.
A MSM has to be defined to model the impacts of the fluid type (A or B) on the V1 failure rate. 
A HMM representation of the pump increases the number of states. The approximation is made through the HMM-P2 model:

$$
\begin{aligned}
& P_{H M M-P 2}= \\
& {\left[\begin{array}{cccc}
9.97 \cdot 10^{-1} & 2.61 \cdot 10^{-3} & 0 & 1.52 \cdot 10^{-4} \\
0 & 9.97 \cdot 10^{-1} & 2.61 \cdot 10^{-3} & 7.99 \cdot 10^{-4} \\
0 & 0 & 9.97 \cdot 10^{-1} & 2.61 \cdot 10^{-3} \\
0 & 0 & 0 & 1
\end{array}\right]}
\end{aligned}
$$

The combination of component states leads to define the MC model of this system over 11 states of the system.

A model of this process is easily realised by means of the DBN depicted in Fig. 7. V1 $(k)$ have three states: Available (o), failure Remains Open (fRO) or failure Remains Closed (fRC) and the P2 $(k)$ have four states $(0, \ldots o 2)$ represent the three degradation states and (f) the failure state. The CPT used to estimate the dynamic behaviour of the components reliability are depicted in Fig. 8. The state probabilities of components at current time step can be easily extracted from the nodes $\mathrm{V} 1(k)$ or $\mathrm{P} 2(k)$. The reliability of the system is computed from the logical combination: System is unavailable if $\mathrm{V} 1=\mathrm{fRC}$ or $\mathrm{P} 2=\mathrm{f}$.

The propagation through the DBN model allows taking into account the dependency between the failure modes and the impact of the environment (fluid A or B) for the computation of the system reliability $\mathrm{R}(k)$.

Inferences are realised thanks to the BayesiaLab software that uses an iterative procedure for DBN (http://www.bayesia.com). BayesiaLab is used to simulate the DBN modelling $\mathrm{R}(k)$ of the system over 1000 time steps, as depicted in Fig. 9. The fluid changes from A to B at time step 300.

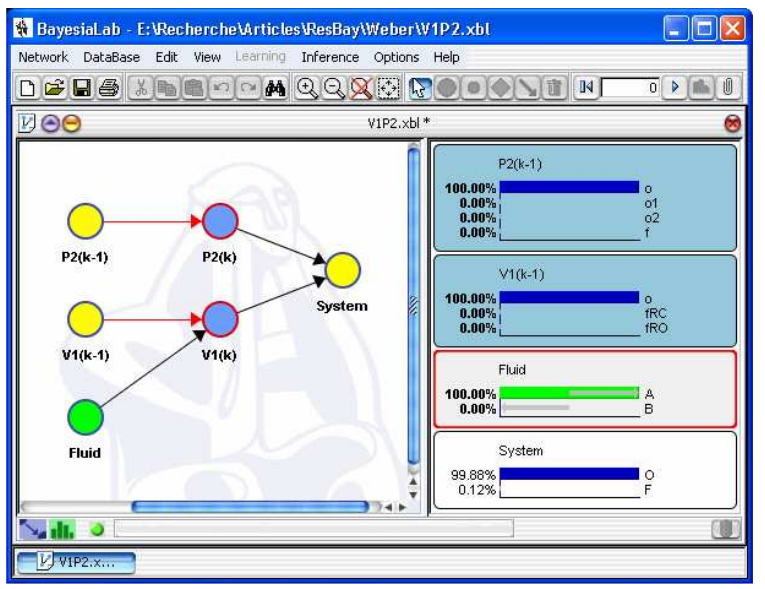

Fig. 7. DBN model of the hydraulic system.

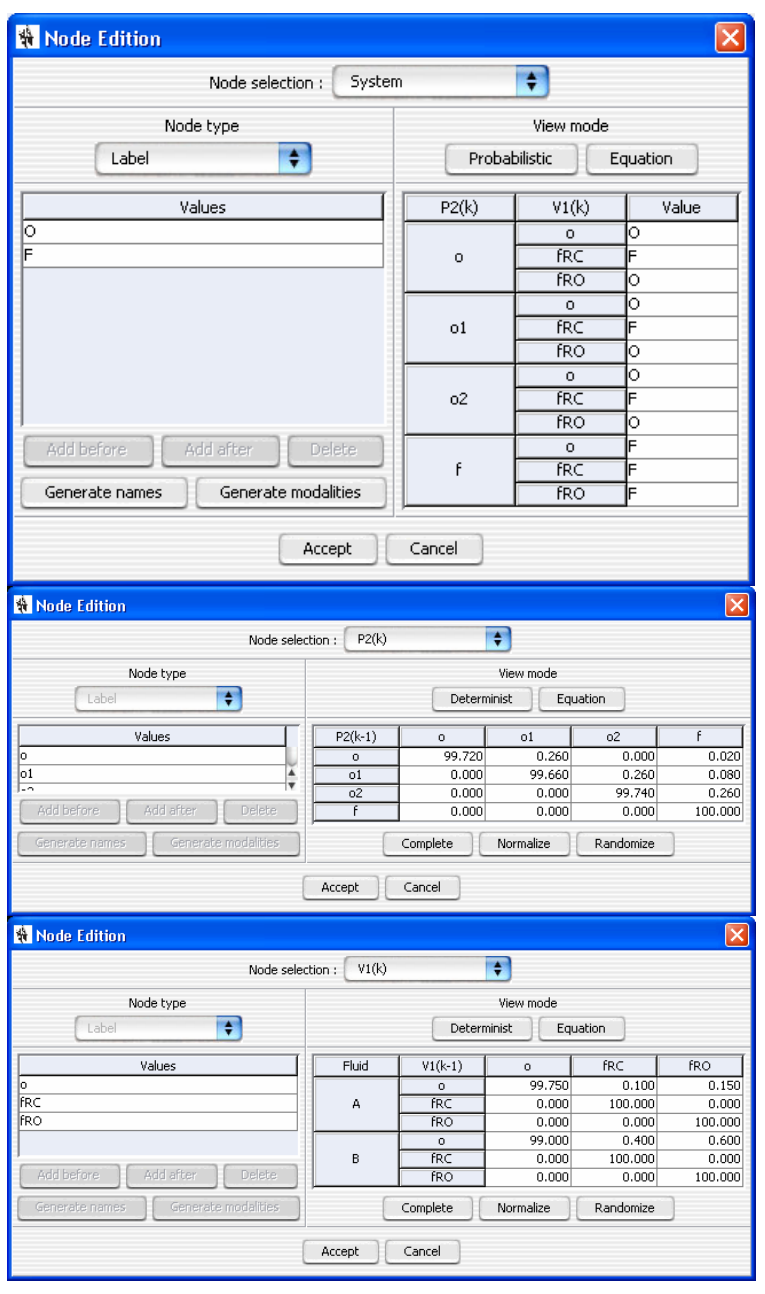

Fig. 8. CPT to model System $(k), \mathrm{P} 2(k)$ and V1( $k)$.

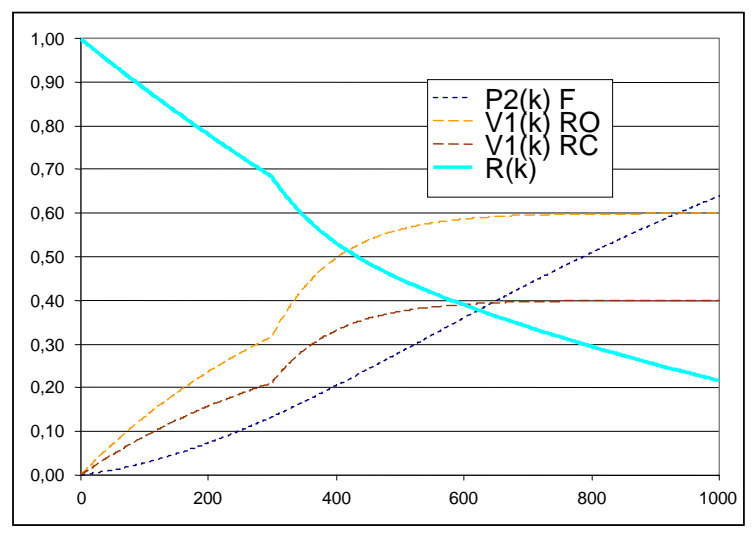

Fig. 9. System reliability estimation.

\section{CONCLUSION AND FURTHER WORK}

The proposed method, based on the DBN theory, easily allows designing DBN structures for the modeling of temporal evolution of complex systems. The correspondence between this DBN representation and $\mathrm{MC}$ is presented and applied to the system reliability estimation.

The proposed method seems to be a good solution to model the reliability of complex systems. Indeed, the number of states needed to model a complex system with MC increases exponentially (a state for each 
combination of elementary states). As the DBNs representation is based on the modelling of process entities, the obtained model is more compact and readable than MC. Furthermore, the dependency between several failure modes of a component and common modes are easily modelled by DBN. This paper shows that DBN represent a very powerful tool for decision-making aid in maintenance.

In future work, in order to improve this modelling technique, we have to define how the learning algorithms of $\mathrm{BN}$ can contribute to model the dynamics of the system reliability and how the parameters behaviour can be then modelled.

\section{REFERENCES}

Ansell, J.I. and M.J. Phillips (1994). Practical methods for reliability data analysis. Oxford University Press Inc., ISBN 0-19-853664-X, New York.

Aven T. and U. Jensen (1999) Stochastic models in reliability. (Applications of mathematics), Springer-Verlag, ISBN 0-387-98633-2, SPIN 10695247, New York.

Bengio Y. (1999). Markovian models for sequential data. Neural Computing Surveys, 2, pp. 129-162.

Bobbio, A., L. Portinale, M. Minichino and E. Ciancamerla (2001). Improving the analysis of dependable systems by mapping fault trees into Bayesian networks. Reliability Engineering and System Safety, 71, pp. 249-260.

Bouissou M. and J.L. Bon (2003). A new formalism that combines advantages of fault-trees and Markov models: Boolean logic driven Markov processes, Reliability Engineering and System Safety, 82 (2), pp 149-163.

Boutillier C., T. Dean, S. Hanks (1999). Decisiontheoretic planning: structural assumptions and computational leverage. Journal of Artificial Intelligence Research, 11, pp. 1-94.

Boyen X. and Koller D. (1998) Tractable for Complex Stochastic Processes. Proceedings of the 14th Annual Conference on Uncertainty in AI (UAI), Madison, Wisconsin, July 1998, pp. 33-42.

Cox D.R. (1962) Renewal theory. Chapman and Hall, ISBN 041220570X, London.

Doucet A., N. De Freitas, K. Murphy, S. Russel, (2000). Rao-Blackwellised particle filtering for Dynamic Bayesian Networks, Proceedings of the 16th Annual Conference on Uncertainty in AI (UAI), pp. 176-183.

Gertsbakh I. (2000). Reliability theory with applications to preventive maintenance. SpringerVerlag (Ed.), ISBN3-540-67275-3, SPIN 10763074, New York.

Hung K. B., S. Venkatesk, G. West (1999). Layered dynamic probabilistic networks for spatiotemporal modelling. Intelligent Data Analysis, $\mathbf{3}$, pp. 339-361.

Jensen, F.V. (1996). An Introduction to Bayesian Networks. (UCL Press (Ed)). London.

Kang, C.W. and M.W. Golay (1999). A Bayesian belief network-based advisory system for operational availability focused diagnosis of complex nuclear power systems. Expert Systems with Applications, 17, pp. 21-32.

Kjaerulff U. (1995). dHugin: a computational system for dynamic time-sliced Bayesian networks. Internationnal journal of forecasting, 11, pp. 89111.

Koller D. and U. Lerner (2001) Sampling in Factored Dynamic Systems. Sequential Monte Carlo Methods in Practice, A. Doucet, J.F.G. de Freitas, and N. Gordon, Eds., Springer-Verlag, pp 445464.

Meshkat L., J.B. Dugan, F.D. Andrews (2002). Dependability analysis of systems with ondemand and active failure modes, using dynamic fault trees. IEEE Trans. On reliability, 51 (2), June.

Padhraic S. (1997). Belief networks, hidden Markov models, and Markov random fields : A unifying view. Pattern Recognition Letters, 18, pp. 12611268.

Torres-Toledano J.G., L.E. Sucar (2003). Bayesian Networks for Reliability Analysis of Complex Systems. Lecture Notes in Computer Science "Progress in Artificial Intelligence IBERAMIA'98, Lisbon, Portugal, Oct. 1998. Proceedings", Springer-Verlag Heidelberg, ISSN: 0302-9743, 1484, Jan 1998, pp 195 - 206.

Weber P., M.C. Suhner and B. Iung (2001). System approach-based Bayesian Network to aid maintenance of manufacturing process. 6th IFAC Symposium on Cost Oriented Automation, Low Cost Automation, Berlin, Germany, 8-9 October, pp. 33-39.

Weber P., L. Jouffe (2003). Reliability modelling with Dynamic Bayesian Networks. 5th IFAC Symposium on Fault Detection, Supervision and Safety of Technical Processes (SAFEPROCESS'03), Washington, D.C., USA, June 9-11.

Welch R. and T. Thelen (2000). Dynamic reliability analysis in an operational context: the Bayesian network perspective. In Dynamic reliability: future directions. Edited by: C. Smidts, J. Devooght and P.E. Labeau, ISBN 0-9652669-3-1, Maryland, USA. 